\title{
Olive Oil: Nutraceutical and Pharmaceutical Food
}

\author{
Esmaeil Ghanbari Shendi ${ }^{*}$ \\ ${ }^{1}$ Hacettepe University, Department of Food Engineering, Ankara, Turkey
}

Corresponding Author: Esmaeil Ghanbari Shendi, PhD of Food Engineering, Hacettepe University, Department of Food Engineering, Ankara 06800, Turkey. Tel: +98-9143110087, Email: esi.1361@gmail.com

Received October 6, 2018; Accepted February 20, 2019; Online Published March 15, 2019

\begin{abstract}
Olive and olive oil is a major part of the Mediterranean diet and breakfast. This tree is a member of Oleaceae family. The genus Olea is containing 2 subgenera Tetrapilus and Olea. The Mediterranean diet that includes up to $40 \%$ of calories as fat, led to a decrease in chronic diseases because of the high oleic acid intake. Olive oil is the oily juice, which is obtained from the olive fruit. It has a characteristic sensory profile and nutrition value. There is a good balance of fatty acids, between saturated, monounsaturated, and polyunsaturated acids in olive oil. This oil is unique among edible and vegetable oils and it can be consumed in the crude form, therefore, its tocopherols and phenolic content is conserved. Olive oil has very valuable minor components, such as phenolic compounds, sterols, vitamin and antioxidants. This study aim to review, some significant pharmaceutical effects and health benefits.

Keywords: Olive Oils, Nutraceutical, Health Benefit, Functional Food

Citation: Ghanbari Shendi E. Olive oil: nutraceutical and pharmaceutical food. Int J Med Rev. 2019;6(1):28-30. doi:10.29252/IJMR-060106.
\end{abstract}

\section{Introduction}

Mediterranean diet which is, including fruits, vegetables, cereals, fish, olives, and olive oil, is the main reason of lower incidence of cardiovascular disease, atherosclerosis, neurodegenerative diseases, and cancers in this region. These functional effects have been attributed to the regular consumption of virgin olive oil (VOO), as the principal source of fat. Olive oil has very valuable minor components such as, phenolic compounds, sterols, vitamin and antioxidants. The chemical composition of olive oil varies, depending on the genetic, geographic and agronomic conditions, harvest time and technological factors.

VOO's main fatty acids are C14:0 (myristic acid), C16:0 (palmitic acid), C16:1 (palmitoleic acid), C17:0 (heptadecanoic acid), C17:1 (cis-10-Heptadecenoic acid), C18:0 (stearic), C18:1n9c (oleic), C18:1n9t (elaidic acid), C18:2n6c (linoleic acid), C18:3n3 (alfa-linolenic acid), C20:0 (arachidic acid), C20:1n9 (cis-11-eicosenoic acid), C22:0 (behenic acid) and C24:0 (lignoceric acid). Monounsaturated fatty acids of VOO are mainly oleic acid and palmitoleic acid, polyunsaturated fatty acids are linoleic acid and linolenic acid, and saturated fatty acids are palmitic acid, stearic acid, arachidonic acid, myristic acid. Minor compounds such as tocopherols ( $\alpha$, $\beta$ and $\gamma$ ), phenolic compounds including apigenin, caffeic acid, gallic acid, luteolin, m-coumaric acid, p-coumaric acid, p-coumaric acid, oleuropein, syringic acid, trans-ferulic acid, vanillic acid, vanillin, tyrosol, 3-hydroxy tyrosol, 3.4 -dihydroxy benzoic acid, 4-hydroxy benzoic acid, 4-hydroxy phenyl acetic acid, ligstroside, lignans have important role in functional properties of VOO. ${ }^{1}$

\section{Phenolic Compounds in Olives and Olive Oils}

Polyphenols (phenolic compounds) are secondary plant metabolites, in greater or lesser amounts in almost all fruit and vegetables. Phenolic compounds more commonly used name "polyphenols" are compounds having phenol functional group. Most of phenolic compounds give the plants their characteristic color of yellow-red-blue tones, as well as flavor components such as bitterness and astringency. Olive is one of the fruits that contain phenolic compounds with high density. Phenolic compounds have distributed in specific proportions, almost in all parts of olive such as leaves, husk, and core; mostly in pulp.

Concentration of the phenolic compounds of VOO depends on climate, soil where olives grow, variety, fruit maturity, harvest method, extraction method, relaxation temperature and time. It affects shelf life and sensory properties of VOO. ${ }^{2}$ Polyphenols with different chemical structures, are extracted from extra VOO. Wide ranges (50-1000 ppm) have been reported for the levels of total polar phenols in olive oils. Common range is between 100 and 300 ppm. Studies on different olive varieties have shown that, some phenolic compounds can be specific for a single variety and also amount of some phenolic compounds have differed among cultivars. In addition, Oleuropein is the major polyphenol glycoside in olive oil, and its amount depends on the cultivar.

The common polyphenols of VOO are aglycons form of secoiridoids. Major secoiridoids in VOO are the dialdehydic form of elenolic acid linked to 3,4-dihydroxyphenyl ethanol (3,4-DHPEA) or $p$-hydroxyphenyl) ethanol ( $p$-HPEA) (3,4-DHPEA-EDA or $p$-HPEA-EDA) and an isomer of the

Copyright (C) 2019 The Author(s). This is an open-access article distributed under the terms of the Creative Commons Attribution License (http:// creativecommons.org/licenses/by/4.0), which permits unrestricted use, distribution, and reproduction in any medium, provided the original work is properly cited. 
oleuropein aglycon (3,4-DHPEA-EA). 3,4-DHPEA and ( $p$-HPEA) are reported as the main phenolic alcohols in VOO; their concentrations are generally low in fresh VOOs, but increases during storage time. Hydrolysis of VOOs' secoiridoids such as 3,4-DHPEA-EDA, p-HPEA-EDA and 3,4-DHPEA-EA is the main reason of this increment. Other phenolic fractions in Extra VOO are luteolin, apigenin, lignans such as (+)-1-acetoxypinoresinol, (+)-1-pinoresinol and (+)-1-hydroxypinoresinol, hydroxy-isochromans such as 1-phenyl-6,7-dihydroxy-isochroman and 1-(3'-methoxy-4'hydroxy) phenyl-6,7-dihydroxy-isochroman. ${ }^{1,2}$

\section{Effect of Polyphenol on the Stability of VOO}

Stability of VOO depends on, polyphenols and tocopherol isomers content. Dialdeydic form of elenolic acid linked to hydroxytyrosol (3,4-DHPEA-EDA), decarboxymethyl oleuropein aglycon and hydroxytyrosol are mainly resistance factors of olive oil to oxidation, but tyrosol, lignans and ligstroside aglycon have weaker antioxidant activity. ${ }^{2}$

\section{Effect of Olive Oil on Cancer}

The anticancer activity of olive oil has been reported in many studies. Bartoli et $\mathrm{al}^{3}$ indicated that, olive oil in dietary prevented the development of aberrant crypt foci and colon carcinomas in rats. These effects have been suggested to be partly mediated through modulation of arachidonic acid metabolism and local PGE synthesis. Same result was obtained for dimethylbenz(a)anthracene-induced mammary carcinogenesis in rats, that olive oil dietary reduce tumor size, incidence and its multiplicity. ${ }^{4}$ Olive oil was shown to induce significant levels of apoptosis (HT29 and Caco-2) and cell differentiation (Caco-2), but did not inhibit proliferation of either cell line. ${ }^{5}$ Other study showed that, an extract from the skin of olive fruits composed of maslinic acid and oleanolic acid, has the cancer chemopreventive activity on colon cancer. $^{6}$

\section{Effect on Coronary Heart Disease}

Prescription of a Mediterranean diet, to patients who have had a myocardial infarction, reduce the risk of recurrence of cardiovascular accident. ${ }^{7}$ Scientific studies approve that, saturated fatty acids are twice as effective at raising blood cholesterollevels as are polyunsaturated, and monounsaturated fatty acids at lowering blood cholesterol. Mono- and polyunsaturated fatty acids have similar effects in cholesterol lowering. In addition, some researchers have shown that, monounsaturated fatty acids decline total blood cholesterol, LDL cholesterol, apolipoprotein B and triglycerides, without any changes in HDL cholesterol and Apo-I plasma levels. ${ }^{8}$ Some researchers ${ }^{9}$ reported that, rats were fed linseed oil, as opposed to olive oil, had lower contents of cholesterol, triglycerides, and phospholipids in plasma and lipoproteins. On the other hand, lipid peroxidation sensitivity of LDL was higher. Polyunsaturated fatty acids decrease blood lipids, ${ }^{10,11}$ but they increase the oxidative susceptibility of LDL. ${ }^{12,13}$

\section{Antioxidant Activity}

Polyphenols have high antioxidant activity. VOO's oxidative stability increase with higher polyphenol content. Hydroxytyrosol as one of the important polyphenols can donate a hydrogen to free radicals. This action resolves its harmful effect. On the other hand, hydroxytyrosol has metal ions chelating ability, that are themselves pro-oxidant agents. ${ }^{14}$ considering the ability of the polyphenols to protect against LDL cholesterol oxidation, some studies indicate that, both hydroxytyrosol and oleuropein inhibit $\mathrm{CuSO} 4$-induced oxidation of LDL which depends on its concentration. Luteolin and lutean aglycon have also protecting effects against LDL oxidation. ${ }^{15,16}$

\section{Effect of Olive Oil on Inflammation}

Scientific research showed that, olive oil polyphenols are mediators of inflammation. Miles et $\mathrm{al}^{17}$ reported that, the different polyphenols of olive oil such as vanillic, p-coumaric, syringic, homovanillic and caffeic acids, kaempferol, oleuropein glycoside, and tyrosol had ability to inhibit the proinflammatory effects of lipopolysaccharide using diluted human blood cultures. They reported that, the polyphenols have varying degrees of inhibiting the cytokines at different levels.

In the present study, oleuropein and caffeic acid decreased the concentration of interleukin-1 $\beta$.

\section{Effect of Olive Oil on Hypertension}

olive oil may decrease blood pressure. A research study showed that, the diet enriched with olive oil decreased the mean blood pressure. ${ }^{18}$ a crossover study in women with hypertension revealed that, olive oil, as opposed to high oleicacid sunflower oil, significantly decreased both systolic and diastolic blood pressure. ${ }^{19}$ Alonso and Martinez-Gonzalez ${ }^{20}$ reported, lower blood pressure among men who consumed more olive oil in their diets, but not in women. Fito et $\mathrm{al}^{21}$ reported that, extra VOO, lowered systolic blood pressure in hypertensive patients more than refined olive oil.

\section{The Effect of Oleuropein on Ischemic Myocardium}

Recently, studies have focused on the development of new methods to limit infarct size and other manifestations of postischemic injury. The gold standard in the treatment of acute myocardial infarction is reperfusion therapy. Restoration of blood flow to ischemic tissue results in a paradoxical phenomenon known as ischemic-reperfusion injury. The potentially harmful aspect of myocardial reperfusion injury, termed lethal reperfusion injury, is defined as myocardial damage due to the restoration of coronary blood flow after potentially fatal ischemic insult. Myocardial injury thus results in cell death in itself. Animal studies have shown that, fatal reperfusion injury is responsible for $50 \%$ of the final infarct size and focused on the prevention of many new strategies. ${ }^{9}$

\section{Conclusions}

Olive oil is a functional food and it has nutraceuticals effects. According to the research results, olive oil has important role on improving of human health. This is attributed to its major and minor compounds. Although, the protective effect of olive oil against cardiovascular diseases is well documented, 
there are few reports on the possible positive effects of olive oil on the gastrointestinal tract. The therapeutic health features of bioactive compounds in olive oil are attributed to the high concentration of monounsaturated fatty acids and polyphenols. Due to its high content of oleic acid, olive oil is known as healthy and safe fat when compared to other edible oils. It can also reduce cardiovascular disease risk due to its useful effect on blood lipid compounds. It can be said that olive oil is not only food, it is also a natural drug without any side effect on human health.

\section{Conflict of Interest Disclosures}

The author declares he has no conflicts of interest.

\section{Reference}

1. Ghanbari Shendi E, Sivri Ozay D, Ozkaya MT, Ustunel NF. Changes occurring in chemical composition and oxidative stability of virgin olive oil during storage. Oilseeds Fats Crops Lipids. 2018;25(6):18. doi:10.1051/ocl/2018052.

2. Ghanbari Shendi E, Sivri Ozay D, Ozkaya MT, Ustunel NF. Determination of some chemical and quality parameters of changes in Turkish Sari Ulak monocultivar extra virgin olive oil during 12 months of storage. Food Sci Technol. 2018;12(3):28-33. doi:10.15673/fst.v12i3.1038.

3. Bartoli R, Fernandez-Banares F, Navarro E, et al. Effect of olive oil on early and late events of colon carcinogenesis in rats: modulation of arachidonic acid metabolism and local prostaglandin E(2) synthesis. Gut. 2000;46(2):191-199. doi:10.1136/gut.46.2.191.

4. Solanas M, Hurtado A, Costa I, et al. Effects of a high olive oil diet on the clinical behavior and histopathological features of rat DMBA-induced mammary tumors compared with a high corn oil diet. Int J Oncol. 2002;21(4):745-753. doi:10.3892/ijo.21.4.745.

5. Llor X, Pons E, Roca A, et al. The effects of fish oil, olive oil, oleic acid and linoleic acid on colorectal neoplastic processes. Clin Nutr. 2003;22(1):71-79. doi:10.1054/clnu.2002.0627.

6. Juan ME, Wenzel U, Ruiz-Gutierrez V, Daniel H, Planas JM. Olive fruit extracts inhibit proliferation and induce apoptosis in HT29 human colon cancer cells. J Nutr. 2006;136(10):2553-2557. doi:10.1093/jn/136.10.2553.

7. Owen RW, Haubner R, Mier W, et al. Isolation, structure elucidation and antioxidant potential of the major phenolic and flavonoid compounds in brined olive drupes. Food Chem Toxicol. 2003;41(5):703-717. doi:10.1016/S0278-6915(03)00011-5.

8. Salvini S, Sera F, Caruso D, et al. Daily consumption of a highphenol extra-virgin olive oil reduces oxidative DNA damage in postmenopausal women. $\mathrm{Br}$ J Nutr. 2006;95(4):742-751. doi:10.1079/BJN20051674.
9. Yellon DM, Hausenloy DJ. Myocardial reperfusion injury. N Engl J Med. 2007;357(11):1121-1135. doi:10.1056/NEJMra071667.

10. Stangl GI, Kirchgessner M, Reichlmayr-Lais AM, Eder K. Serum lipids and lipoproteins from rats fed different dietary oils. J Anim Physiol Anim Nutr. 1994;71(1-5):87-97. doi:10.1111/j.1439-0396.1994. tb00343.x.

11. Balasubramaniam S, Simons LA, Chang S, Hickie JB. Reduction in plasma cholesterol and increase in biliary cholesterol by a diet rich in n-3 fatty acids in the rat. J Lipid Res. 1985;26(6):684-689.

12. Scaccini C, Nardini $M$, D'Aquino $M$, Gentili V, Di Felice $M$, Tomassi G. Effect of dietary oils on lipid peroxidation and on antioxidant parameters of rat plasma and lipoprotein fractions. J Lipid Res. 1992;33(5):627-633.

13. Parthasarathy S, Khoo JC, Miller E, Barnett J, Witztum JL, Steinberg D. Low density lipoprotein rich in oleic acid is protected against oxidative modification: implications for dietary prevention of atherosclerosis. Proc Natl Acad Sci U S A. 1990;87(10):38943898. doi:10.1073/pnas.87.10.3894.

14. Angerosa F, Di Giacinto L. Oxidation des huiles d'olive vierges par le métaux: manganése et nickel, Note 1 (Metal-induced oxidation of virgin olive oils: manganese and nickel. Note 1).. Rev Fr Corps Gras. 1993;40(1-2):41-48.

15. Visioli F, Bellomo G, Montedoro G, Galli C. Low density lipoprotein oxidation is inhibited in vitro by olive oil constituents. Atherosclerosis. 1995;117(1):25-32. doi:10.1016/00219150(95)05546-9.

16. Visioli F, Galli C. Oleuropein protects low density lipoprotein from oxidation. Life Sci. 1994;55(24):1965-1971. doi:10.1016/00243205(94)00529-X.

17. Miles EA, Zoubouli P, Calder PC. Differential anti-inflammatory effects of phenolic compounds from extra virgin olive oil identified in human whole blood cultures. Nutrition. 2005;21(3):389-394. doi:10.1016/j.nut.2004.06.031.

18. Lahoz C, Alonso R, Porres A, Mata P. Las Dietas Enriquecidas en Ácidos Gracos Monoinsaturadoesy Ácidos Gracos Poliinsaturado Omega 3 Disminuyen la Presion Artersial, sin Modificar la Concentración de Insulina Plasmática en Sujetos Sanos. Med Clin (Barc). 1999;112:133-137.

19. Ruiz-Gutierrez V, Muriana FJ, Guerrero A, Cert AM, Villar J. Plasma lipids, erythrocyte membrane lipids and blood pressure of hypertensive women after ingestion of dietary oleic acid from two different sources. J Hypertens. 1996;14(12):1483-1490. doi:10.1097/00004872-199612000-00016.

20. Alonso A, Martinez-Gonzalez MA. Olive oil consumption and reduced incidence of hypertension: the SUN study. Lipids. 2004;39(12):1233-1238. doi:10.1007/s11745-004-1352-x.

21. Fito $M$, Cladellas $M$, de la Torre $R$, et al. Antioxidant effect of virgin olive oil in patients with stable coronary heart disease: a randomized, crossover, controlled, clinical trial. Atherosclerosis. 2005;181(1):149-158. doi:10.1016/j.atherosclerosis.2004.12.036. 